\title{
Ear, Nose and Throat Approach in Autoimmune Blistering Diseases
}

\author{
Ismail Cem Temel ${ }^{1}$, Asli Bilgic-Temel ${ }^{2 *}$ and Dedee Frances Murrell ${ }^{2,3}$ \\ ${ }^{1}$ Sydney ENT Clinic, Sydney, Australia \\ ${ }^{2}$ Department of Dermatology, St George Hospital, UNSW, Australia \\ ${ }^{3}$ Faculty of Medicine, University of New South Wales, Australia
}

*Corresponding author: Asli Bilgic-Temel Department of Dermatology, St George Hospital, UNSW, Australia.

Received Date: October 08, 2018

Published Date: October 30, 2018

\begin{abstract}
Autoimmune blistering diseases (AIBD) are a group of rare blistering disorders of the skin and mucous membranes due to autoantibodies directed against specific proteins. Involvement of mucosal areas in AIBD significantly increases patient morbidity and mortality. Ear, nose and throat (ENT) examination should be a routine procedure to evaluate mucosal involvement in every AIBD. Patients should be asked whether they had oropharyngeal, ear, nose and throat symptoms and all patients should be evaluated for ENT manifestations by direct and endoscopic examination even in patients without symptoms.
\end{abstract}

Keywords: Autoimmune blistering diseases; Ear; Nose; Throat; Mucosal involvement

Abbreviations: AIBD: Autoimmune Blistering Diseases; IEBD: Intraepidermal Blistering Diseases; SEBD: Subepidermal Blistering Diseases; PV: Pemphigus Vulgaris; PF: Pemphigus Foliaceus; PNP: Paraneoplastic Pemphigus; BP: Bullous Pemphigoid; MMP: Mucous Membrane Pemphigoid; ENT: Ear, Nose and Throat; PDAI:Pemphigus Disease Area Index; ABSIS: Autoimmune Bullous Skin Disorder Intensity Score; DIF: Direct Immunofluorescence Microscopy; ELISA: Enzyme-linked Immunosorbent Assay

\section{Introduction}

Autoimmune blistering diseases (AIBD) are a group of rare blistering disorders of the skin and mucous membranes due to autoantibodies directed against specific proteins. However, they cause significant morbidity and mortality. AIBD could be divided into 2 subgroups: 1 . Intraepidermal blistering diseases (IEBD), 2. Subepidermal blistering diseases (SEBD) [1]

IEBD encompasses pemphigus vulgaris (PV), pemphigus foliaceus (PF) and paraneoplastic pemphigus (PNP) as the main subtypes. PV is the major type and it is most commonly seen between the ages of 40 and 50 years. It usually starts as erosions in the oral mucosa, and then continues with blistering and erosive skin lesions. Other commonly involved areas include the pharynx, larynx, nasal mucosa and oesophagus. Its incidence is estimated to be two new patients per 1 million inhabitants per year in Central and Western Europe. However, it is more commonly seen in Mediterranean and Middle Eastern countries. PNP is a paraneoplastic blistering disease characterized by cheilitis and/or ulcerative stomatitis, persisting painful erosions of mucosa and cutaneous polymorphic involvement (graft-versus-host disease-like, erythema multiformelike, bullous pemphigoid- like or pemphigus vulgaris-like eruption). It usually presents with severe mucosal involvement including the pharyngeal, laryngeal, ocular and genital mucosal surfaces. Its prognosis is poor because of concomitant malignancy. On the other hand, PF does not affect the mucosal surfaces [2].

Bullous pemphigoid is the main AIBD of the subepidermal blistering group. It is the most common AIBD in developed countries and typically seen in people over 70 years old. Although it might affect mucosal areas, it is not as common as seen with PV [3]. On the other hand, mucous membrane pemphigoid (MMP) mainly affects the mucosal surfaces of oropharynx, larynx, nose, eyes, oesophagus and genitals. It usually results in tissue destruction (airway loss, blindness, etc.) and functional limitations (malnutrition, loss of teeth, etc.) because of chronic mucosal inflammation and scarring [4]. 
We aimed to summarise general recommendations and approaches to ENT examination and procedures in AIBD.

\section{Ear, nose and throat involvement}

Involvement of mucosal areas in AIBD significantly increases patient morbidity and mortality. PV in the IEBD group and MMP in the SEBD are the most prominent and common ones affecting the mucosal surfaces (Figure 1-3). However, others might also cause mucosal lesions. Ear, nose and throat (ENT) examination should be a routine procedure to evaluate mucosal involvement in every AIBD. Patients should be asked whether they have oropharyngeal, ear, nose and throat symptoms and all patients should also be evaluated for ENT manifestations by direct and endoscopic examination, even if they do not have any symptoms [5].

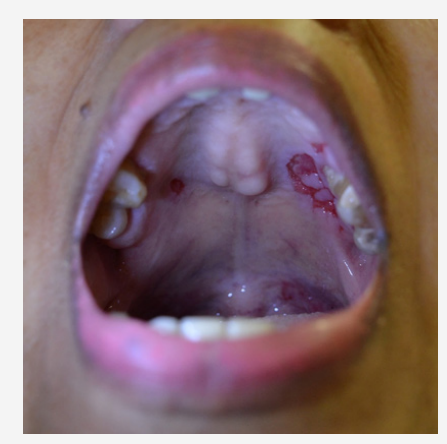

Figure 1: Erosions on the hard and soft palate in a pemphigus vulgaris patient

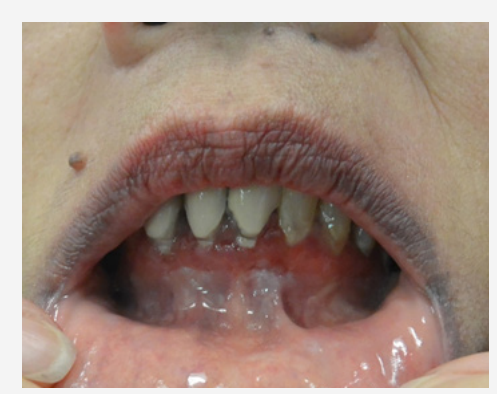

Figure 2: Erosive gingivitis on the lower gingiva in pemphigus vulgaris

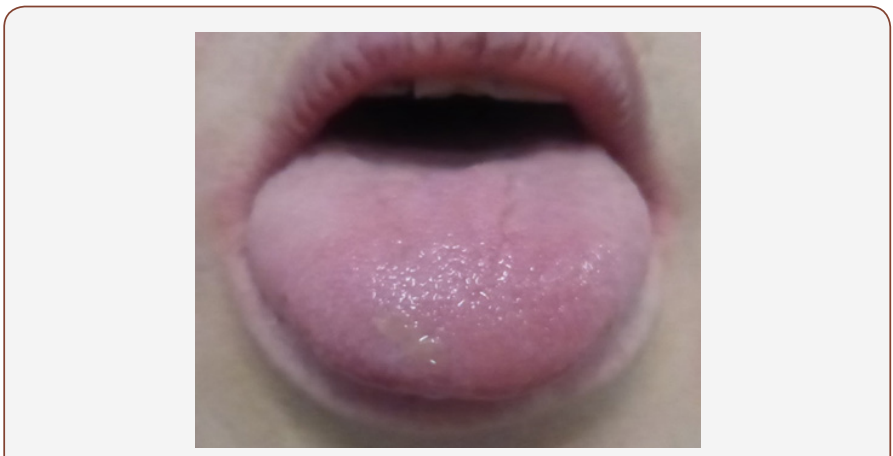

Figure 3: Blister on the tongue in pemphigus vulgaris.

The frequency of ENT involvement was studied and found to be more frequent than expected, especially in PV, by several groups [5-11]. Our recent study involving 50 patients with PV additionally suggested that the severity of ENT involvement in PV was significantly associated with ENT symptoms and endoscopic findings, especially in cases of severe disease (Pemphigus Disease Area Index (PDAI) $\geq 15$ and/or Autoimmune Bullous Skin Disorder Intensity Score (ABSIS) $\geq 17$ ] [5]. However, apart from severe and scarring forms of AIBD, even mild mucosal involvement can cause discomfort, bleeding and erosions on the mucosae.

Thus, ENT surgeons should apply a specific examination and procedural approach in the care of these patients.

\section{Procedural approach}

Patients with AIBD are ideally followed in specific clinics where a multidisciplinary approach is available. However, patients with AIBD are often first seen by an ENT specialist in a local hospital. Thus, every ENT surgeon should be aware of the essential steps in the diagnosis and management of such patients.

\section{Diagnosis}

\section{Biopsy}

Clinical features, compatible light microscopy findings and positive direct immunofluorescence microscopy (DIF) findings and/ or positive specific Enzyme-linked Immunosorbent Assay (ELISA) autoantibodies are necessary for the diagnosis of AIBD [2-3]. Specimens for light microscopy studies should be taken from early bullae arising (from the edge of the lesion, including the lesional mucosa and the normal mucosa around it) on erythematous/ normal mucosa or skin and placed in formalin solution with care not to leave the epidermis from the dermis [12].

The most critical test in the diagnosis of AIBD is DIF. Its positivity is essential for the diagnosis of AIBD according to wellestablished guidelines [2,3]. The biopsy specimen for DIF should be obtained from perilesional mucosa (avoid the blistered mucosa or erosion), and for transportation, skin biopsy specimens for DIF studies should be put into Michel's fixative or simply $0.9 \% \mathrm{NaCl}$ solution $[2,13]$.

\section{General recommendations}

Thereare some recommendations suggested in the management of patients with AIBD, established in the guidelines and in expert opinions $[2,3,14,15]$ (Table 1$)$.

Table 1: summarises these recommendations.

\section{Inform the patients and their families about the disease, its nature, available treatments, possible side effects of each treatment and complications}

Inform the patients about the need to follow their regular appointments to monitor disease activity, treatment tolerance and/or any side effects.

Inform the patients about potential triggers such as drugs, physical trauma and operations.

Add dietary supplements in patients with malnutrition.

Although there is insufficient evidence in terms of restrictions about diet, patients can be informed about avoiding high amounts of certain foods (such as garlic,onion, chive, and leek) which are thought to be triggers of AIBD (especially for pemphigus) (15).

Patients should be vaccinated against seasonal influenza, H1N1 and pneumococci most appropriately before any therapy. However,

if patients are using immunosuppressive therapies (high dose corticosteroids, azathioprine, rituximab, etc.), live attenuated vaccines are contraindicated.

Patients should be informed about specific AIBD referral centres. 


\section{ENT examination and clinical approach}

There are certain steps that can be taken by practitioners to optimise the ENT examination. These steps are summarised in [2,3,14-18] (Table 2).

Table 2: Recommendations for ENT examination and clinical approach.

Question recent drug-intake as it is well-known that some drugs (D-penicillamine, angiotensin-converting enzyme inhibitors, betablockers, etc.) can trigger AIBD $(15,16)$.

Document the patient's general condition and comorbidities in the file.

Examine all mucous membranes to determine the degree of mucosal damage and functional impairment (dysphagia, dysphonia, hoarseness, etc.)

Do not cause any mechanical trauma by direct pressure and/or friction on the skin and mucosal surfaces, as trauma can create new blisters and erosions, especially in active disease due to the Nikolsky phenomenon (slight rubbing of the skin/mucosa results in exfoliation of the outer layer of the skin/mucosa) $(17,18)$.

Avoid rigid endoscopes and nasogastric tubes and use flexible ones

Lubrication of necessary equipment is also very important to decrease the trauma risk.

Drain new blisters by a sterile needle. Overlying mucosa should be left intact to provide a biologic cover of the erosion.

A dermatologist familiar with AIBD should also be involved in the process of diagnosis and the treatment plan for these patients.

\section{During ENT operations}

Moreover, there are important points to consider before, during and after the ENT operations. These bullet points are lined up in $[2,3,14]$ (Table 3).

Table 3: Recommendations for ENT operations.

Preoperative assessment by practitioners (ENT surgeon and dermatologist) is important to plan the procedure according to the patients' needs and condition.

Document all topical and systemic therapies (even intermittent ones, such as rituximab, intravenous immunoglobulin, etc.) in the file.

Examine previous operations to anticipate the possible risks (such as any complication in previous surgeries, etc.

Explain anticipated risks of the operation to the patients and/or care givers.

Patients should continue their usual skin care habits, such as emollients and antiseptic washes, and topical therapies (topical steroid or antibiotics) during inpatient units before and after surgery.

Be careful during transportation for operations.

Lubricate the equipment (including laryngoscopes, endotracheal and nasogastric tubes)

Pulse oximeter probes, electrocardiogram and electrocautery electrodes should be applied without adhesive tapes especially in patients with active disease (patients with blisters and erosions).

\begin{tabular}{|c|}
\hline Avoid hard plastic oxygen masks \\
\hline Protect the eyes during surgery. Eyes should be closed and covered \\
appropriately.
\end{tabular}

\section{Monitoring the disease}

Patients should be monitored in specialised AIBD centres according to several measures such as clinical condition, the severity and disease progress during treatment and the therapeutic choice (monitoring, tolerance, adverse-effects) [2,3].

\section{Conclusion}

Every ENT specialist should be aware of AIBD as they can be the first practitioner evaluating these patients. Thus, awareness can provide early diagnosis which results in better control of the disease and reduces morbidity and mortality. Skin and mucous membrane fragility of these patients need a safe and effective procedural approach during ENT mucosal examination and/or procedures.

\section{Acknowledgements}

Dr. Ismail Cem Temel is a consultant ENT surgeon at Seydisehir State Hospital, Konya, Turkey. He is currently working as an Observer at Sydney ENT Clinic, Sydney, Australia.

Dr. Asli Bilgic-Temel is a consultant Dermatologist and now working as a Visiting Dermatology Fellow at St. George Hospital, UNSW, Sydney, Australia. She is the recipient of the Turkish Dermatology Society - Prof. Dr. Hulusi Behcet (Long-term Research) Scholarship in 2018.

\section{Conflict of Interest}

None.

\section{References}

1. Schmidt E, Borradori L, Joly P (2015) Epidemiology of Autoimmune Bullous Diseases. Blistering Diseases: Clinical Features, Pathogenesis, Treatment. Dédée F (Ed.), Murrell First Edition. Springer-Verlag Berlin Heidelberg, Netherlands, pp. 251-263.

2. Hertl M, Jedlickova H, Karpati S, Marinovic B, Uzun S, et al. (2015) Pemphigus. S2 Guideline for diagnosis and treatment--guided by the European Dermatology Forum (EDF) in cooperation with the European Academy of Dermatology and Venereology (EADV). J Eur Acad Dermatol Venereol 29(3): 405-414.

3. Feliciani C, Joly P, Jonkman MF, Zambruno G, Zillikens D, et al. (2015) Management of bullous pemphigoid: the European Dermatology Forum consensus in collaboration with the European Academy of Dermatology and Venereology. Br J Dermatol 172(4): 867-877.

4. Amber KT, Murrell DF, Schmidt E, Joly P, Borradori L. (2018) Autoimmune Subepidermal Bullous Diseases of the Skin and Mucosae: Clinical Features, Diagnosis, and Management. Clin Rev Allergy Immunol 54(1): 26-51.

5. Bilgiç Temel A, Temel I.C, Bostancı Toptaş A, Turhan M, Bozkurt S, et al. (2016) The effect of endoscopic ear, nose and throat examination on pemphigus severity scoring systems (poster presentation). Pathogenesis of Pemphigus and Pemphigoid, Munich, Germany.

6. Fawzy MM, Hegazy RA, Abdel Fattah AF. (2013) Ear, nose, and throat involvement in Egyptian patients with pemphigus vulgaris: a step towards a better management. Int J Dermatol 52 (10): 1268-1273.

7. Robati RM, Rahmati-Roodsari M, Dabir-Moghaddam P, Farnaghi A, Mahboobi-rad F, et al. (2012) Mucosal manifestations of pemphigus vulgaris in ear, nose, and throat; before and after treatment. J Am Acad Dermatol 67 (6): 249-252.

8. Fernández S, España A, Navedo M, Barona L (2012) Study of oral, ear, nose and throat involvement in pemphigus vulgaris by endoscopic examination. Br J Dermatol. 167: 1011-1016. 
9. Kavala M, Altıntaş S, Kocatürk E, Zindancı I, Can B, et al. (2011) Ear, nose and throat involvement in patients with pemphigus vulgaris: correlation with severity, phenotype and disease activity. J Eur Acad Dermatol Venereol. 25 (11): 1324-1327.

10. España A, Fernández S, del Olmo J, Marquina M, Pretel M, et al. (2007) Ear, nose and throat manifestations in pemphigus vulgaris. Br J Dermatol156 (4): 733-737.

11. Hale EK, Bystryn JC. (2001) Laryngeal and nasal involvement in pemphigus vulgaris. J Am Acad Dermatol 44 (4): 609-611.

12. Erkin G (2011) The Histopathological Diagnosis of Autoimmune Bullous Diseases. Turkderm. 45 Suppl 1: 26-30.

13. Uzun S (2011) Immunofluoresecence Findings in the Diagnosis of Autoimmune Bullous Diseases. Turkderm. 45 Suppl 1: 31-5.
14. Uzun S (2012) Current Management of Pemphigus. Turk J Dermatol 6: 91-101.

15. Tavakolpour S (2018 ) Pemphigus trigger factors: special focus on pemphigus vulgaris and pemphigus foliaceus. Arch Dermatol Res 310(2): 95-106

16. Stavropoulos PG, Soura E, Antoniou C (2014) Drug-induced pemphigoid: a review of the literature. J Eur Acad Dermatol Venereol 28(9): 11331140.

17. Urbano FL. (2001) Nikolsky's sign in autoimmune skin disorders. Hosp Physician 37: 23-4.

18. Uzun S, Durdu M (2006) The specificity and sensitivity of Nikolskiy sign in the diagnosis of pemphigus. J Am Acad Dermatol 54(3): 411-415. 